\title{
Research on On-line Temperature Monitoring System of Power Cable
}

\author{
Cheng Dawei ${ }^{1, \text { a) }}$, Zhao Dongxu ${ }^{2, b)}$ and $Y u M^{2}{ }^{1, c)}$ \\ ${ }^{1}$ The Electric Power Research Institute of Liaoning Province Power Company \\ ${ }^{2}$ Liaoning Electric Power Co., Ltd. \\ a)chengdawei@dbdky.com \\ b)zjy1106@sina.com \\ c)yumiao@dbdky..com
}

\begin{abstract}
Power cable temperature monitoring system is based on the cable installation, cable surface temperature and load current and cable thermal characteristics of the environment, the use of real-time calculation program to calculate the operating temperature of the conductor to ensure that the power cable running in a rated temperature range and to ensure The cable will not overheat and early aging conditions. Aiming at the function requirement of the on-line temperature monitoring system of the cable, the modular design method is used to divide the system design task into two functional modules, which are designed separately. However, these two functional modules are self-contained but belong to the whole system Of the sub-module.
\end{abstract}

Key words: Power Cable, Condition monitoring system; Senior Application System;

\section{INTRODUCTION}

With the rapid development of China's national economy, urban construction and modernization of enterprises increased, as the lifeblood of the national economy met an unprecedented challenge. In order to meet the demand of power supply, the proportion of power cables in urban power grid is more and more, which leads to more and more management and maintenance of power cables. Especially in cities with rapid economic development such as Beijing and Shanghai, the urban power grids show high load density and high load growth [1]

Which requires the use of new high-voltage, high-capacity power transmission and distribution equipment in-depth near the center of the city center load power. The use of high voltage, large cross-section cable to meet the requirements of large transmission capacity, but the city control and line-intensive so that the addition of new power transmission facilities is extremely difficult. As the laying of new power facilities face a lot of pressure, so that the existing power lines to enhance the safety of cable transmission capacity becomes very important. In order to improve the transmission capacity of power cables, to ensure the operation environment of power cable facilities and the main body of power cable facilities, the existing cable monitoring system must be improved to supervise the new operation state of power cables. Therefore, to maximize the use of power cable transmission capacity, is to meet the high load density and cable in the central city of power supply requirements, but also to create a conservation-oriented society and effective measures. The substation in the city center is dense and the project is complicated. The underground cables are widely distributed and the distance is long. In the event of accident, the cable will be seriously damaged. In order to effectively guarantee the 
transmission capacity of the power channel and improve the channel resource utilization, the safe operation of the cable As well as maintenance more worthy of further study.

\section{RELATED RESEARCH}

The on-line monitoring technology of power cable line is the first technical measure to find the location of local hot spot of power cable, detect the insulating state of running line and calculate the carrying capacity of conductor. Including cable fault detection, cable insulation testing, current detection and temperature monitoring. While the temperature of the conductor can be the most direct response to the normal operation of the cable. Since the conductor does not have the desired electrical conductivity when the cable is running, the resistance-induced loss is proportional to the square of the current flowing through the conductor, and this loss is manifested by heat. The insulation of the cable allows the temperature to determine the maximum current through the conductor, the conductor operating temperature can only be estimated from the jacket temperature or surface temperature. [2].

The domestic calculation of the conductor temperature rise mainly focuses on the analysis of the cable characteristics under the linear thermal environment. The on-line monitoring of the cable is also limited to the measurement of the cable current and cable surface temperature or the insulation temperature without further analysis of the cable conductor Of the temperature rise. In this way, the concept of the actual temperature of the conductor is blurred when monitoring the operation of the cable. Rough evaluation of the operating current of the cable from the measured cable surface or insulation temperature will result in wasteful use of the cable. The foreign cable monitoring system is more emphasis on safety enhance the cable carrying capacity, so the conductor real-time temperature monitoring more experienced in 2003 Ander and 2004 de Wild proposed dynamic feedback simulation model. For the current domestic and international temperature collection technology are the following[3]:

\section{Point temperature sensing technology}

Point temperature sensing technology is the scene in the temperature set according to the needs of a number of point sensors (such as thermocouple, thermal resistance and thermal relays, etc.) to measure the temperature of a specific point of space and the corresponding cable through the temperature analog Or over-limit signal upload to the host for processing technology. Mainly based on the temperature sensor or thermocouple measurement technology, the temperature sensor or thermocouple placed in the line prone to failure, such as the cable terminal and the middle connector, or cable local heat source to monitor the temperature of these parts. This method of investment, operation is simple, and can only get the local temperature line.

\section{On - line Monitoring Technology of Power Cable Based on Thermal Effect}

The current research is mainly the use of infrared technology, such as infrared thermal imager. The cable surface temperature measurement, the core temperature inversion calculation, to achieve non-contact temperature of the cable core, real-time online diagnosis. Although this method can make up for the lack of local cable temperature measurement, but the infrared effect of the thermal effect of the temperature measured by the object emissivity, environmental and aerosol greater impact

\section{Linear Temperature Sensing Technology}

Mainly in the temperature field according to the need to lay one or more by the special temperature conductivity of the material made of temperature sensing cable, the cable along the cable placed in parallel, or tied to the cable outer sheath to measure a certain area Space temperature of segment. When the cable temperature exceeds the fixed temperature value, the sensing cable is short-circuited and an alarm signal is sent. Disadvantage is a destructive alarm, alarm temperature is fixed, fault signal is incomplete. And can not measure the actual temperature value of the cable; because of the large number of cables, the system installation and maintenance work is not convenient enough, easy to damage the equipment; can not be early fault prediction, can not real-time display of measured values, no temperature trend analysis[4] 


\section{Fiber Optic Sensor Technology}

Fiber-optic sensor is a new type of sensor based on optical fiber developed in recent years. Fiber optic sensors use light as a carrier of sensitive information, using optical fibers as a medium for transmitting sensitive information. So it has the characteristics of optical measurement: good electrical insulation, strong anti-electromagnetic interference, high sensitivity, easy to realize long-distance monitoring of the measured signal. The principle of optical fiber sensor is to measure the state into a measurable optical signal device. It uses the time difference between the incident light and the backscattered light and the light propagation speed in the fiber, can calculate the distance of the different scattering point from the incident end, so the fiber can be obtained along almost continuous temperature

\section{SYSTEM OVERALL DESIGN}

In this chapter, the overall scheme of the system will be designed, the implementation of the scheme will be demonstrated, and several key technologies to be discussed will be discussed in order to select the appropriate MCU, temperature sensor, host computer and peripheral components in the subsequent chapters. Specific hardware design[5].

In addition, the overall design of the program should also consider the following points:

1) System can be convenient, intuitive and effective monitoring of cable temperature changes;

2) The system must be able to collect the temperature value of the cable joint in real time and process it quickly.

3) The system can display the data processing results to the user, but also be able to store the results to facilitate future

4) Than research.

5) System's economy, reliability requirements;

6) 5 temperature measurement points, to be easy to expand;

7) Easy operation and maintenance;

\section{The hardware design of monitoring terminal}

System hardware design is the main consideration is the overall structure of the system is reasonable, simple and cost-effective selection. Therefore, the main indicators in terms of hardware is practical, the subject of the monitoring terminal installed in the cable connector and the cable is easy to heat the site for measuring the cable temperature, the temperature of the environment and the hum[6].

\section{System working sub-station data processing flow}

In order to reduce the energy consumption of the system, the software design of each working substation is very important. The simple program can effectively save the running time of the microcomputer, thus reduce the energy consumption; make the CPU work in the power-down or idle mode. Can effectively reduce power consumption. Power-down mode power consumption than idle mode is lower, but because of power-down mode requires a hardware reset wake-up. So the design is selected CPU idle mode.

\section{The slave station receives the data processing flow}

Each working substation has a local address $\mathrm{M}$, an address $\mathrm{A}$, a destination address $\mathrm{D}$, a source address $\mathrm{S}$, and a relay address $\mathrm{N}$ of the nRF 905. Data format to be sent after processing, the host receives a limited cache, in order to avoid multiple operating points when the transmission of conflict, the sender to contact. Depending on the direction in which data is transmitted, each operating point can determine the address code $\mathrm{A}$ at the receiving end. When the working point receives a data packetr[6].

Unpacking, starting the decision, and deciding what to do with the packet.

1) When $\mathrm{M}=\mathrm{D}$, that is, the current operating point address is the receiving address, then the received data packet returns ACK, and processing data. Otherwise (2);

2) to determine the relationship between $\mathrm{D}$ and $\mathrm{S}$ to determine the direction of transmission; 
3) to determine the relationship between $M$ and $N$, to determine the sender address $A$. If $D>S, M>N$, the forwarding address is $A$ +1 ; if $\mathrm{D}<\mathrm{S}$, the forwarding address is $\mathrm{A}+1$ when $\mathrm{M}<\mathrm{N}$. Forwarding, the current operating point also need to relay work

The point returns an ACK. The current working point address $\mathrm{M}$ is used instead of the original relay point address $\mathrm{N}$ in the forwarded packet.

\section{Sub-station to send data flow}

Transmit mode, the PTR8000 can be completed within the wireless system automatically power on, data packet and packet transmission (GFSK, Manchester encoding). In the design process to pay attention to the address set TX address must be the same chip with the address of the receiver, [7].

Wireless transmitter program flow can be divided into the following steps:

1) Configure the PTR8000 module in power-down or standby mode;

2) Set the interface speed by protocol or MCU;

3) Start transmission by setting TRX CE and TXEN;

4) When TRX CE is low, the transmission is ended and the PTR8000 is in standby mode

\section{CONCLUSION}

The power cable on-line temperature monitoring system developed in this paper can not only monitor the conductor temperature of the cable, but also can provide reference for the operation efficiency of the cable and play an active role in the prevention of fire.

Make full use of the information (online, offline, inspection, etc.), with many artificial intelligence techniques (neural network, fuzzy mathematics, case-based reasoning and rough set), the evaluation of equipment service life and reliability analysis of the introduction of advanced application system, with the foundation of state maintenance function, but also has the characteristics of center of maintenance on the reliability;

\section{REFERENCES}

1. Fred Steennis; Paul Wagenaars; Guarding MV cables on-line: With travelling wave based temperature monitoring, fault location, PD location and PD related remaining life aspects,[J] IEEE Transactions on Dielectrics and Electrical Insulation,2016 page 34-39

2. Bo Zhu; Xinlao Wei; Song Wang; Ruihai Li, On-line monitoring method for long distance power cable insulation [J]. IEEE Journals \& Magazines, 2016,Pages: 70 - 76

3. Xiaosheng Peng; Chengke Zhou; Donald M. Hepburn; Martin D. Judd; W. H. Siew, Application of K-Means method to pattern recognition in on-line cable partial discharge monitoring[J], IEEE Transactions on Dielectrics and Electrical Insulation2013, Pages: 754 - 761

4. STATE GRID, 《Technical guide for on-line monitoring system of transformation equipment》, Bei Jing, General Office of the State Grid,2011-04-28

5. Y. Tian; P. L. Lewin; J. S. Wilkinson; G. Schroeder; S. J. Sutton; S. G. Swingler, An improved optically based PD detection system for continuous on-line monitoring of HV cables[J], IEEE Transactions on Dielectrics and Electrical Insulation,2005, Pages: 1222 - 1234,

6. STATE GRID ,Q / GDW 679-2011, 《Function specifications for integrated supervision and control system of smart substation》， Bei Jing, General Office of the State Grid, 2012-02-07

7. CAO Yang,Yao Jiangguo,Yang ShengChun,JIANG Hai,Gao ZhiYuan,Latest Advancements of Smart Grid Core Standard IEC 61970[J]. .Automation of Electric Power System.2011.9.31-36 